\title{
Esquistosomiasis: una parasitosis urinaria cada vez más frecuente
}

\author{
López López AI*, Cao Avellaneda E*, Prieto González A*, Ferri Ñíguez B**, Maluff Torres A*, \\ Pérez Albacete $\mathrm{M}^{*}$.
}

*Servicio de Urología. **Servicio de Anatomía Patológica Hospital Universitario Virgen de la Arrixaca. Murcia.

Actas Urol Esp. 2007;31(8):915-918

\section{RESUMEN}

ESQUISTOSOMIASIS: UNA PARASITOSIS URINARIA CADA VEZ MÁS FRECUENTE

Las parasitosis urinarias, muy infrecuentes hasta la fecha en nuestro medio, están siendo diagnosticadas cada vez con mayor frecuencia debido a los fenómenos migratorios.

Presentamos un nuevo caso de esquistosomiasis urinaria y realizamos una revisión de la literatura en relación a su diagnóstico y tratamiento.

Paciente subsahariano de 28 años que consulta por hematuria y síndrome miccional de 1 año de evolución.

La cistoscopia muestra características granulaciones blanquecinas pequeñas en la mucosa vesical y el estudio anatomopatológico de la biopsia fría de la mucosa vesical confirma la existencia de huevos y parásitos adultos.

A pesar de la infrecuencia de la esquistosomiasis en nuestro medio y en relación al aumento de los fenómenos migatrorios poblacionales, debemos incluir esta afectación urinaria en el diagnostico diferencial de la hematuria monosintomática.

Palabras clave: Esquistosomiasis urinaria. Bilharziosis

\section{ABSTRACT \\ SCHISTOSOMIASIS: NOT AN UNCOMMON PARASITOSIS IN EUROPE}

Despite the fact that urinary parasitosis are very unusual diseases in our place, their frequency is growing up because of migration movements.

Clinical case: A 28 years old subsaharian patient presented with haematuria and low urinary symptoms.

The cystoscopic shows small white round granulate in bladder mucosa and anatomopathologic study informed about eggs an adult parasites on bladder biopsy.

Despite of the infrequency of schistosomiasis in our country and because of migration movements we must include this disorder in monosimptomatic haematuria differential diagnosis.

Keywords: Urinary schistosomiasis. Urinary bilhaziosis.

$\mathrm{L}$ a esquistosomiasis urogenital es una de las muchas parasitosis padecen los habitantes de los países pobres. En los países del África Subsahariana constituye uno de los principales problemas de Salud Pública afectando principalmente a la infancia.

Ésta enfermedad, como algunas otras parasitosis, está aumentando su incidencia en Europa como consecuencia de la mayor movilidad poblacional por lo que debe ser incluida por los urólogos europeos en sus algoritmos diagnósticos.

\section{CASO CLÍNICO}

Paciente varón de 28 años de edad, oriundo de Mali, país del que partió cinco años atrás. Actualmente reside en España desde hace 2 
años, donde llegó atravesando Costa de Marfil, Camerún, Togo, Gambia, Zaire, Benin, Nigeria, Argelia y Marruecos. Consulta por cuadro de hematuria intermitente de un año de evolución acompañada de intenso síndrome miccional. Recuerda cuadro de dolor abdominal, unos meses atrás, fundamentalmente localizado en hipogastrio sin nauseas ni vómitos pero con sensación distérmica y escalofríos. También refiere dolor testicular que se irradia a región anal desde hace varios días, por el que ha sido tratado con Ciprofloxacino y AINES sin encontrar mejoría. Niega relaciones sexuales sin protección.

A la exploración física el abdomen es blando y depresible no doloroso a la palpación sin masas ni megalias. Peristaltismo normal. Testes y epidídimos normales. Ausencia de secreción uretral purulenta. Adenopatías bilaterales en cadenas inguinales, blandas y móviles de características inflamatorias.

El sedimento urinario es normal y la analítica general muestra leucopenia de 2560 leucocitos con fórmula leucocitaria normal. El paciente aporta una analítica antigua, de cuando consultó por dolor abdominal, que evidencia leucocitosis con neutrofilia (36208 leucocitos con $34 \% \mathrm{~N})$. Se solicita serología de anticuerpos para Schistosoma que resulta positiva a títulos de $1 / 64$. La Rx de abdomen muestra calcificaciones vesicales lineales (Fig. 1) y la UIV evidencia una estenosis ureteral distal izquierda (Fig. 2). La ecografia no muestra hallazgos significativos y el examen de la orina en fresco no fue concluyente. Realizamos una cistoscopia observando múltiples lesiones redondeadas, pequeñas y blanquecinas en la totalidad de la mucosa, de las que se toman muestras de biopsia fría.

El resultado del estudio anatomopatológico de las mismas destaca la presencia de estructuras redondeadas basófilas, con cutícula periférica, compatibles con schistosomas. No se identifica en su periferia respuesta histiocitaria o granulomatosa. (Figs. 3 y 4).

El paciente se trató con Praziquantel en dosis única $40 \mathrm{mg} / \mathrm{kg}$ de peso en monodosis. Evoluciona favorablemente 3 meses después y sigue revisiones ambulatorias.

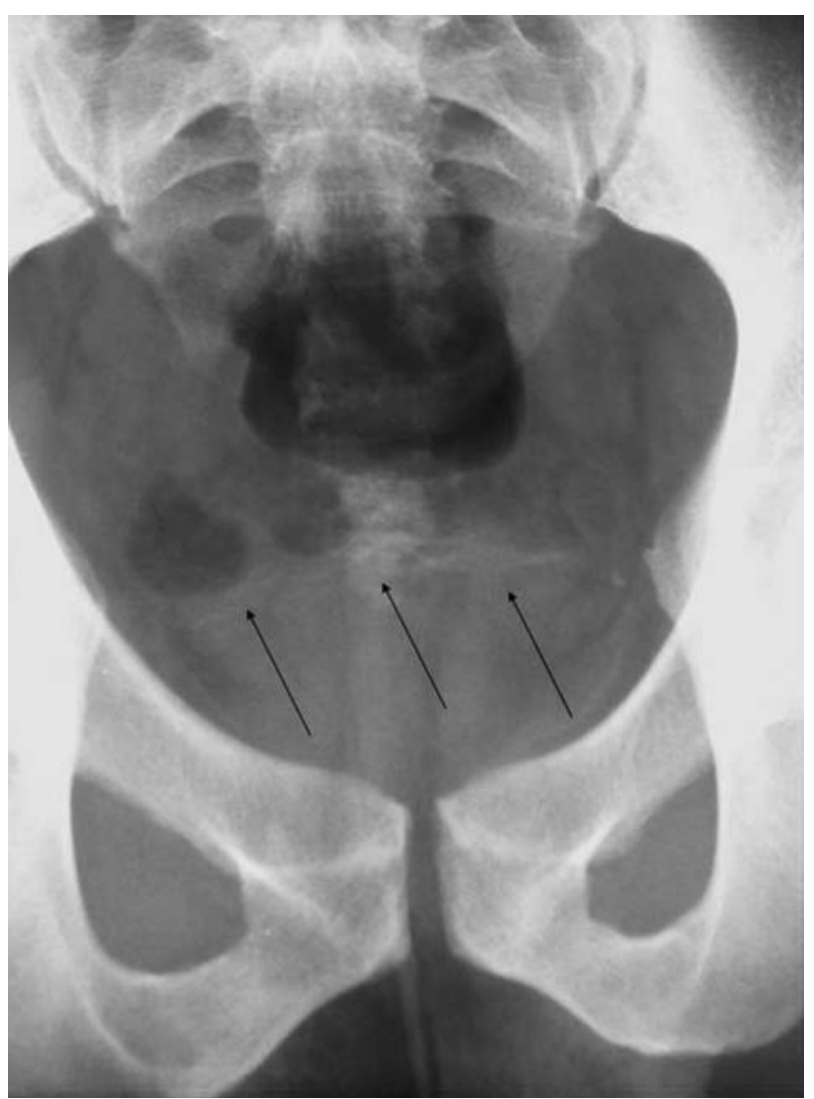

FIGURA 1. Imágenes de calcificaciones vesicales lineales en radiografia simple de abdomen.

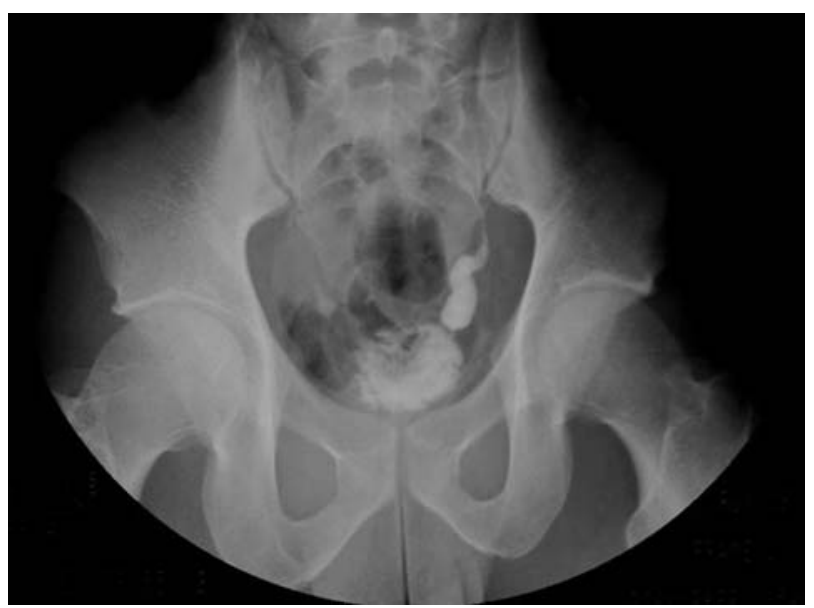

FIGURA 2. Imagen de urografia intravenosa en la que se observa estenosis ureteral distal izquierda.

\section{DISCUSIŌN}

La esquistosomiasis o bilharziasis urinaria, es uno de los mayores problemas de Salud Pública que padecen los países pobres ${ }^{1}$. El Schistosoma haematobium es el causante de esta patología en más de 100 millones de personas en el mundo ${ }^{2}$, 


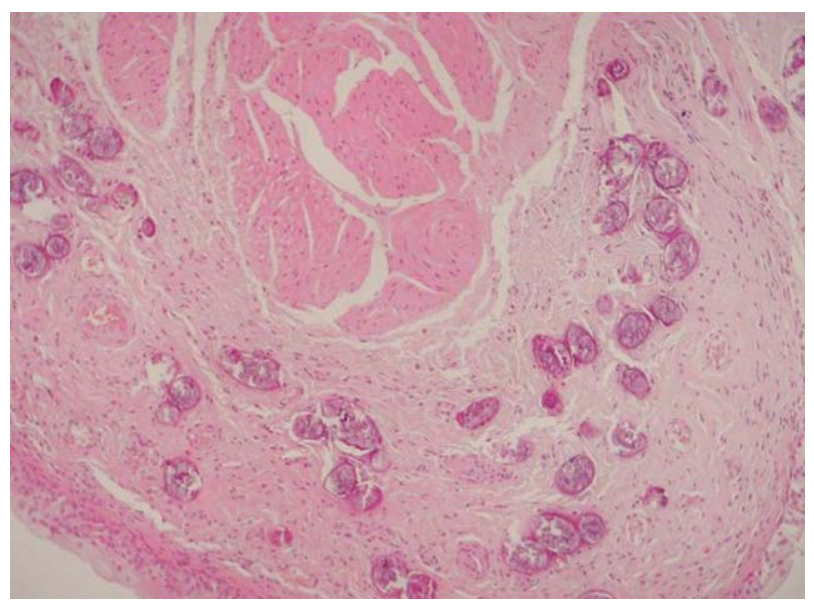

FIGURA 3. Biopsia vesical que muestra a nivel de la lámina propia abundantes huevos de Schistosoma haematobium sin reacción inflamatoria-granulomatosa periférica.

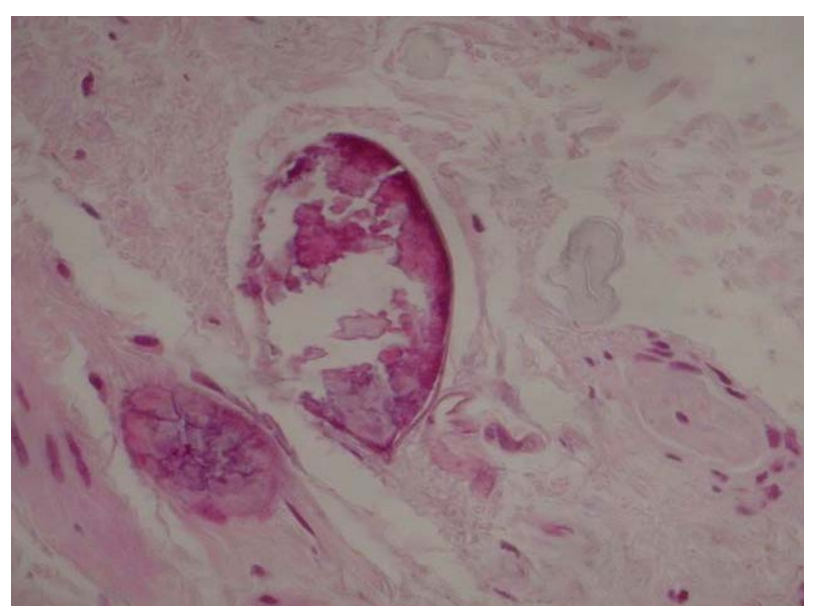

FIGURA 4. Detalle a gran aumento de Schistosoma haematobium con espina terminal.

afectando fundamentalmente a la población rural del África Subsahariana y los países de la cuenca del Nilo. Esta afección, que ha sido muy infrecuente en nuestro entorno, ha visto incrementada su incidencia debido a la emigración y la mayor facilidad para viajar a los países tradicionalmente endémicos ${ }^{3}$.

La Esquistosomiasis es un conjunto de infecciones parasitarias con formas clínicas agudas y crónicas causadas por trematodos del género Schistosoma. Son cinco las especies comúnmente implicadas en las infecciones humanas (S. japonicum, S. mansoni, S. haematobium, S. mekongi y S. intercalatum), pero los trastornos urinarios obedecen fundamentalmente a la infección por Schistosoma haematobium ${ }^{4}$.
El ciclo de estos parásitos es indirecto y en él el hombre es el huésped definitivo. Los gusanos adultos emiten sus huevos en los vasos sanguíneos adyacentes a la vejiga; los huevos atraviesan la pared de este órgano, caen a su luz y se eliminan por la orina. En el agua dulce se abren y de ellos sale un embrión, llamado miracidio, que nada hacia un molusco, el bulinus, cuyos tegumentos atraviesa para acabar transformándose en las formas larvarias del parásito, las furocercarias, que de nuevo atravesarán los tegumentos del este molusco para salir de él y nadar en el agua dulce. Otra vez en agua dulce la mayoría de las larvas desaparecerán y sólo algunas conseguirán fijarse la a piel de un nuevo huésped definitivo-otro ser humano- para continuar el ciclo. El ser humano se infecta al bañarse en agua dulce, sobre todo durante las horas de calor y soleadas. Las larvas se fijan a la piel y las cabezas la atraviesan penetrando en el torrente circulatorio. En los vasos hepáticos mudarán al estadio adulto y tras la fecundación las hembras alcanzarán, a través del sistema portal, los plexos hemorroidales, vesicales y vaginales donde depositarán los huevos.

Las manifestaciones clínicas cambian en función de la fase del ciclo de infestación. En la zona de penetración de las larvas son características unas places eritematosas y pruriginosas que desaparecen en pocos días. Durante la migración de las larvas por el organismo -fase de invasiónlas sustancias antigénicas producidas por los parásitos son las responsables de las manifestaciones clínicas, sin embargo, estos síntomas-síntomas inespecíficos como dolor de cabeza, fiebre o malestar general- son menos intensos que los producidos por otras especies ya que el S. haematobium se adapta muy bien al ser humano. En la esquistosomiasis urogenital la clínica es más llamativa en la fase crónica por la presencia de los huevos en la pared de la vejiga, uréter y órganos genitales y su reacción inflamatoria granulomatosa acompañante ${ }^{2,4}$. La fase de estado se produce a los dos meses de la infección y se caracteriza por hematuria discontínua de sangre roja brillante que puede acompañarse de disuria y polaquiuria como en el caso de nuestro paciente. Si la evolución de la enfermedad es prolongada se pueden producir complicaciones como engrosa- 
miento parietal y fibrosis vesical, estenosis ureteral o hidronefrosis y en escasas ocasiones insuficiencia renal. La esquistosomiasis ha sido relacionada con el carcinoma epidermoide vesical ${ }^{5,6}$. En relación a la afectación genital en el varón la esquistosomiasis puede producir uretritis, epidimiditis y hematospermia y en la mujer infertilidad y aumento en la frecuencia de embarazos ectópicos ${ }^{2}$.

El diagnóstico se basa en la detección de huevos del parásito en orina. La recogida de orina debe realizarse entre las 11 am y las $2 \mathrm{pm}$ momento en el que, al parecer, la excrección del parásito es máxima. El recuento de la cantidad de huevos en la muestra de orina permite evaluar la severidad de la infección, que se considerará severa cuando sea superior a 400 huevos por cada $100 \mathrm{ml}$ de orina ${ }^{2} . \mathrm{El}$ análisis del sedimento urinario nos permitirá comprobar la existencia de hematuria o proteinuria. La citología urinaria puede revelar la presencia de células tumorales relacionadas con la infección por Esquistosoma.

En relación a las pruebas de imagen una radiografía simple de abdomen puede mostrar calcificaciones lineales finas más o menos continuas en la pared de la vejiga, una calcificación total de la pared vesical o "vejiga en porcelana"o una característica calcificación en el segmento distal de los uréteres. La urografía intravenosa puede contribuir a localizar estenosis ureterales o ser útil para diagnosticar la hidronefrosis producida por este tipo de parasitosis. Han sido definidos una serie de hallazgos ecográficos típicos e indicativos de esta enfermedad como pared vesical mamelonada y gruesa, pólipos vesicales sesiles o pediculados, o dilatación pielocalicial con cavidades irregulares, sin embargo la limitación fundamental de esta prueba es su escasa sensibilidad en las fases más precoces de la enferme$\operatorname{dad}^{7}$.

La utilidad fundamental de la cistoscopia se halla en los casos en los que las técnicas de imagen o el recuento de huevos en la orina no es concluyente ya que permite obtener biopsia fría de la mucosa vesical así como para descartar degeneración neoplásica. Las lesiones que pueden observarse microscópicamente varían en función del desarrollo de los granulomas inflamatorios alrededor de los huevos. A veces pueden observarse granulaciones blanquecinas pequeñas, como azúcar en polvo, lesiones parecidas a granos de arroz, pólipos o lesiones ulcerativas de distinta profundidad.

El tratamiento de elección en la actualidad es el Praziquantel $40 \mathrm{mg} / \mathrm{kg}$ de peso en monodosis o en dos dosis de $20 \mathrm{mg} / \mathrm{Kg}$ separadas 12 horas que normalmente es bien tolerado ${ }^{2}$.

El conocimiento de determinadas entidades, que siendo infrecuentes en nuestro medio van aumentando su incidencia debido a la mayor movilidad de las personas, parece ser ineludible y para ello debemos recordar incluir en nuestra anamnesis datos que nos orienten hacia el diagnóstico de las mismas.

\section{REFERENCIAS}

1. Chistulo L, Engels D, Montresor A, Savioli L The global status of schistosomiasis and its control. Acta Trop. 2000 Oct 23;77(1):41-51.

2. Bichler KH, Savatovsky I, Naber KG, Bischop MC, Bjerklund-Johansen TE, et al. EAU Guidelines for the Management of Urogenital Schistosomiasis. Eur Urol. 2006 Jun;49(6):998-1003. Epub 2006 Feb 28.

3. Corachan M, Almeda J, Vinuesa T, Valls ME, Mejías T, Jou $\mathrm{P}$ et al. Schistosomiasis imported by Spanish travellers: clinic-epidemiologic study of 80 cases. Med Clin (Barc). 1997 May 17;108(19):721-725.

4. Navarro Cabañas G, García Sánchez N, Rubio Rubio R, Izaguirre Zugazaga C, Clavel Parrilla A, Seral García C. Esquistosomiasis urogenital: un diagnóstico sencillo. An Pediatr (Barc). 2006 Mar;64(3):290-291.

5. Badawi AF, Mostafa MH, Probert A, O'Connor PJ. Role of schistosomiasis in human bladder cancer: evidence of association, aetiological factors, and basic mechanisms of carcinogenesis. Eur J Cancer Prev. 1995 Feb;4(1):45-59.

6. Khurana S, Dubey ML, Malla N. Association of parasitic infections and cancers. Indian J Med Microbiol. 2005 Apr; 23(2):74-79.

7. Keita AD, Sangho H, Sacko M, Diarra Z, Simaga SY, Traore I. Prevalence of schistomasiasis lesions detected by ultrasonography in children in Molodo, Mali. Gastroenterol Clin Biol. 2005 Jun-Jul;29(6-7):652-625.

Correspondencia autora: Dra. A.I. López López

Servicio de Urología. Hospital Universitario Virgen de la Arrixaca.

Ctra. Madrid-Cartagena s/n - 30120 El Palmar (Murcia)

Telf.: 968369278

E-mail autora: llanais@hotmail.com

Información artículo: Nota clínica

Trabajo recibido: mayo 2006

Trabajo aceptado: junio 2006 